\title{
The VODKA sensor: a bio-inspired hyperacute optical position sensing device
}

\author{
Lubin Kerhuel, Stéphane Viollet and Nicolas Franceschini
}

\begin{abstract}
We have designed and built a simple optical sensor called Vibrating Optical Device for the Kontrol of Autonomous robots (VODKA), that was inspired by the "tremor" eye movements observed in many vertebrate and invertebrate animals. In the initial version presented here, the sensor relies on the repetitive micro-translation of a pair of photoreceptors set behind a small lens, and on the processing designed to locate a target from the two photoreceptor signals. The VODKA sensor, in which retinal micro-scanning movements are performed via a small piezo-bender actuator driven at a frequency of $40 \mathrm{~Hz}$, was found to be able to locate a contrasting edge with an outstandingly high resolution 900-fold greater than its static resolution (which is constrained by the interreceptor angle), regardless of the scanning law imposed on the retina. Hyperacuity is thus obtained at a very low cost, thus opening new vistas for the accurate visuo-motor control of robotic platforms. As an example, the sensor was mounted onto a miniature aerial robot that became able to track a moving target accurately by exploiting the robot's uncontrolled random vibrations as the source of its ocular microscanning movement. The simplicity, small size, low mass and low power consumption of this optical sensor make it highly suitable for many applications in the fields of metrology, astronomy, robotics, automotive, and aerospace engineering. The basic operating principle may also shed new light on the whys and wherefores of the tremor eye movements occurring in both animals and humans.
\end{abstract}

Index Terms-Astronomy, hyperacuity, metrology, Micro Aerial Vehicle (MAV), optical sensor, photodiode, photoreceptor, Position Sensing Device (PSD), robotics, Unmanned Aerial Vehicle (UAV), vibration, vision.

\section{INTRODUCTION}

$\mathrm{T}^{\mathrm{H}}$ E human visual system is able to detect an object subtending an angle of only a few secs of arc between two objects, which is an angle much smaller than the interreceptor angle. This property has been called hyperacuity [1]. Another type of hyperacuity is the ability of a natural or artificial optical sensor to locate a contrasting object with a higher resolution than the theoretical (static) resolution. Although the mechanisms involved in the various kinds of hyperacuity have not yet been completely elucidated, retinal micro-movements seem to play an important role [2], [3]. Hennig et al. have shown, for example, that the tiny eye movements called tremor can improve the spatial resolution and induce hyperacuity in the case of vernier

L.Kerhuel was with the Institute of Movement Sciences, Biorobotics team, National Center for Scientific Research, University of the Mediterranean, F13288 Marseille cedex 09, France. He is now with MOVEA S.A., F-38000 Grenoble, France (email: lubin@kerhuel.eu).

S.Viollet and N.Franceschini are with the Institute of Movement Sciences, Biorobotics team, National Center for Scientific Research, University of the Mediterranean, F-13288 Marseille cedex 09, France (email: stephane.viollet@univmed.fr, nicolas.franceschini@univmed.fr).

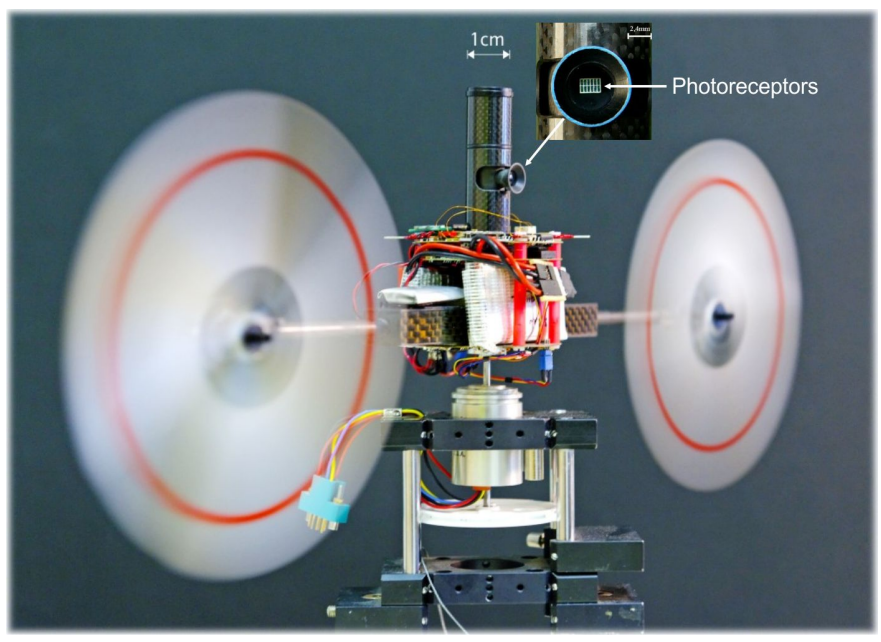

Fig. 1. OSCAR 2 robot equipped with the hyperacute VODKA sensor. The 100-gram aerial robot, which is usually suspended from the ceiling with a long $100 \mu m$ nylon wire [7]-[9] controls its yaw on the basis of what it sees. Here it was mounted on the axis of a frictionless resolver that allowed its heading to be accurately monitored. The twin-engine robot orients itself by driving its two propellers differentially. The inset shows the robot's retina (after the lens has been removed), which can be subjected to repetitive microtranslations at a frequency of $40 \mathrm{~Hz}$ by means of a piezo-bender (see Fig.2). VODKA's retina is composed of an Ic-Haus LS2C package comprising 6 vertically elongated photoreceptors with integrated amplifiers. The VODKA vibrating sensor actually uses only the two central photoreceptors (2 pixels, as sketched in Fig. 2).

stimuli [4]. Chen et al. have noted that visual scanning may also provide a useful means of enhancing the visual acuity of subjects wearing visual prostheses [5]. In a more general framework, Altes reported that jitter sensitivity in echolocation, differential pitch sensitivity in audition, and vernier acuity in vision seem to be based on similar principles [6].

However, hyperacuity can also exist without requiring any visual scanning movements. Krotkov studied hyperacuity by combining classical information theory with image processing algorithms [10]. Riley et al. recently implemented a hyperacute visual sensing machine, using a complex optical arrangement and high-sensitivity optical sensors [11]. Brückner et al. assessed the hyperacuity of an artificial compound eye in terms of its ability to locate a point source or an edge [12]. The robustness of this visual sensors' performance with respect to contrast and the distance from the object targeted has not been addressed so far, however. In many cases, the hyperacuity of artificial visual sensors results from rather complex processing 
steps. In the field of image processing, for example,there has been a considerable amount of work on edge detection and edge localization over the past decades. Several authors have used discrete subpixel image displacement methods (called « dithering ») to enhance the resolution of large image sensors, but this is at the cost of large memory resources [13]-[16].

In the optical sensor presented here, hyperacuity in edge localization results from an active visual process whereby specific continuous micro-movements are applied to an artificial retina. This approach was inspired by the retinal micro-movements observed in the eyes of many invertebrates (Arachnids [17], [18], Molluscs [19], and Insects [20]). The retinal micro-scanning which occurs in the housefly's eye [20] has already served as a biological model for miniature visual sensors [21]-[24], the efficiency of which has been assessed onboard various mobile robots [9], [21], [25]-[27]. Retinal scanning in jumping spiders [17] has also served as a biological model for a visual sensor [28]. Prokopowicz and Cooper designed an active imaging system based on human eye tremor, which led them to the concept of dynamic retina [29] whereas Hongler et al. used vibration noise to improve dramatically edge detection in an image [30].

Here we focus on a novel optical position sensing device called VODKA, which is endowed with hyperacuity. Contrary to previous studies [9], [22], [26], [27], [31], in which hyperacute target location performance required a specific micro-scanning law imposed on the sensor's retina, VODKA can deal with any scanning law and even exploit random movements caused by uncontrolled vibrations of the platform supporting the eye.

Section II describes the model for the VODKA optical system presented in section III and explains how the VODKA sensor works. In section IV, we describe how changes in several parameters can affect the sensor's static characterictics. In section $\mathrm{V}$,the noteworthy features of the sensor are discussed. In section VI, two experiments are described which show the high accuracy of VODKA in terms of its target localization performance. VODKA's responses were found to depend quasi-linearly on the target's bearings, showing a relatively high robustness to distance and contrast. Lastly, it was established that VODKA shows an outstanding degree of hyperacuity, with the ability to locate a target about 900 times more accurately than might be expected, given the interreceptor angle.

\section{Modeling the Micro-Scanning Sensor VODKA}

\section{A. Optics}

The sensor optics include two photodiodes (Ic-Haus LS2C shown in Fig. 1) placed behind a lens (diameter $D=5 \mathrm{~mm}$, focal length $f=8.5 \mathrm{~mm}$ ) and driven in small translation by a piezo bender actuator (Physik Instrumente PL128), as shown in Fig.2. A bell-shaped directivity function simulating the angular sensitivity function of the fly's photoreceptors [32][36] was obtained here by slightly defocusing the lens (actually reducing the distance between the lens and the retina). The

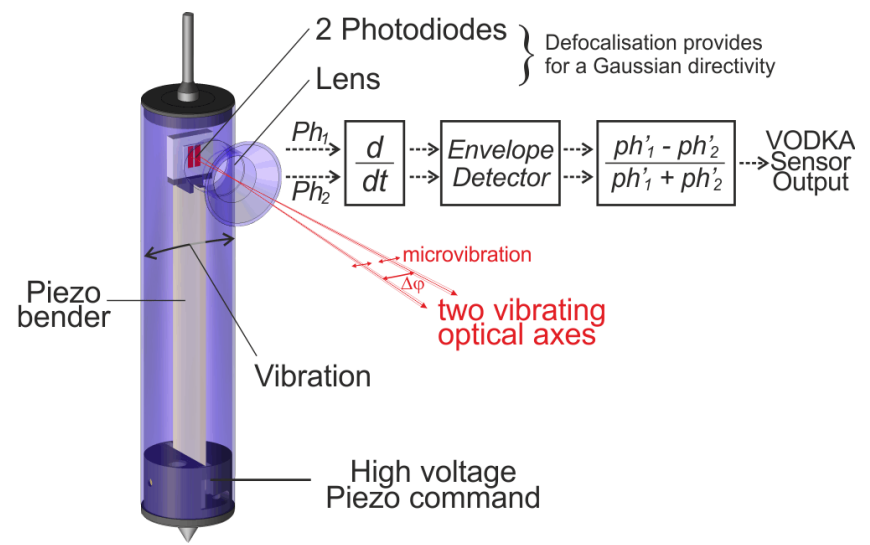

Fig. 2. CAD and sketch diagram of the VODKA sensor. The two photoreceptors are moved back and forth by a piezo-bender located inside the vertical eye tube, making their optical axes jointly scan a fraction of a degree of the environment. The VODKA sensor's output is simply the relative difference between the two filtered photoreceptor signals $P h_{1}^{\prime}$ and $P h_{2}^{\prime}$.

Gaussian-like directivity of each of the two VODKA's photoreceptors can be defined by the parameter denoted $\Delta \rho$, which was defined as the Full Width at Half Maximum (FWHM) (see the directivity functions on the left part of Fig.3). The directivity function $s(\Psi)$ of a photoreceptor is therefore given by:

$$
s(\Psi)=\frac{2 \sqrt{\pi \ln (2)}}{\pi \Delta \rho} e^{-4 \ln (2) \frac{\Psi^{2}}{\Delta \rho^{2}}}
$$

where $\Psi$ is the angle between the photoreceptor optical axis and that of a point light source.

\section{B. Temporal Output Signal of a Photoreceptor}

The output signal of a photoreceptor placed in front of a contrasting feature (an edge or a bar) is the convolution of the projected light intensity with the directivity function $s(\Psi)$.

When a vertical edge is placed at an angular position $\Psi_{c}$ within the Field of View (FOV) of a photoreceptor, its output signal resulting from the convolution of the edge image with the directivity function $s(\Psi)$ is defined as follows:

$$
\begin{aligned}
\operatorname{Ph}\left(\Psi_{c}\right) & =k\left(\frac{1}{2}+\int_{-\infty}^{\Psi_{c}} s(\Psi) d \Psi\right) \\
& =\frac{k}{2}\left(1+\operatorname{erf}\left(\frac{2 \sqrt{\ln (2)} * \Psi_{c}}{\Delta \rho}\right)\right)
\end{aligned}
$$

where the factor $k$ depends on both the contrast and the lighting conditions and erf is the so-called error function defined by:

$$
\operatorname{erf}(\Psi)=\frac{2}{\sqrt{\pi}} \int_{0}^{\Psi} e^{-x^{2}} d x
$$

When a vertical bar of finite width $L$ is encountered, the photoreceptor's output signal resulting from the convolution 


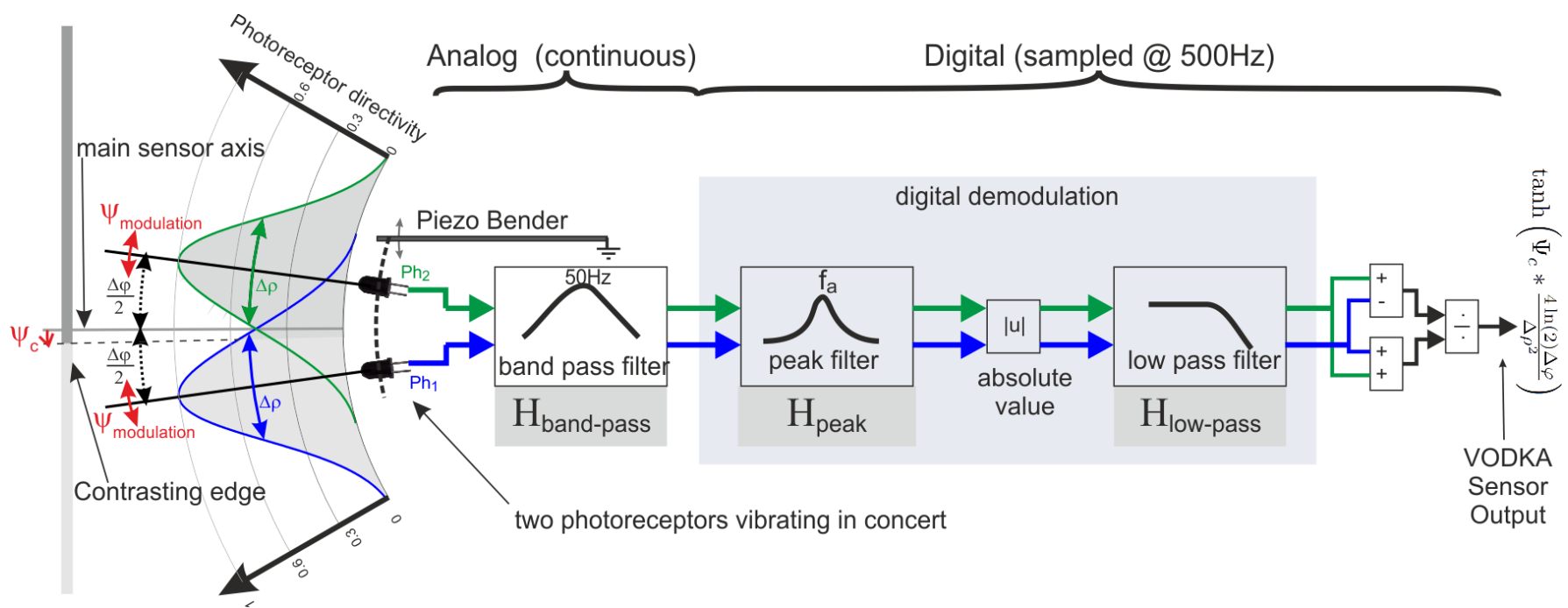

Fig. 3. Sketch diagram of the VODKA sensor. The left part shows the Gaussian angular sensitivity of each of the two photoreceptors placed in front of a contrasting edge. Mechanical vibration is applied to the two photoreceptors, causing their optical axes to rotate jointly. A band-pass filter acting as a pseudo derivative filter extracts the signals generated in each photoreceptor by the relatively high frequency vibration $\left(f_{a}=40 \mathrm{~Hz}\right)$. An optional peak filter removes all frequencies not associated with the vibration. A demodulator extracts the envelope of the signal. The relative difference between the two signals is computed as $\left(P h_{1}^{\prime}-P h_{2}^{\prime}\right) /\left(P h_{1}^{\prime}+P h_{2}^{\prime}\right)$ to yield the VODKA sensor output.

of the bar image with the directivity function $s(\Psi)$ is defined as follows:

$P h\left(\Psi_{c}\right)=\frac{k}{2}\left(\operatorname{erf}\left(\frac{2 \sqrt{\ln (2)}\left(\Psi_{c}+\frac{L}{2}\right)}{\Delta \rho}\right)-\operatorname{erf}\left(\frac{2 \sqrt{\ln (2)}\left(\Psi_{c}-\frac{L}{2}\right)}{\Delta \rho}\right)\right)(4)$

\section{PRINCIPLE OF THE VODKA SENSOR}

The VODKA sensor is designed to estimate the azimuthal position of a vertically oriented contrasting target. Here we take the case of a sensor measuring the angular position of a vertical edge or bar.

The optical assembly comprising the two photoreceptors separates their optical axes by a constant angle denoted $\Delta \varphi$ (see Fig.2 \& Fig.3), which is called the interreceptor angle. The VODKA sensor's main axis is defined as the bisector of these two axes. Each of the two photoreceptors' output signals, which are denoted $P h_{1}$ and $P h_{2}$, depends on the angle between its optical axis and the target (the edge or the center of the bar). Let us take $\Psi_{c}$ to denote the angular position of the visual target relative to the main VODKA axes. The combined vibration of the two optical axes adds a high frequency modulation denoted $\Psi_{\text {mod }}$. The two photoreceptors' output signals can therefore be written as follows:

$$
\begin{aligned}
& P h_{1}(\Psi(t))=P h\left(\Psi_{c}(t)+\Psi_{\text {mod }}(t)-\frac{\Delta \varphi}{2}\right) \\
& P h_{2}(\Psi(t))=P h\left(\Psi_{c}(t)+\Psi_{\text {mod }}(t)+\frac{\Delta \varphi}{2}\right)
\end{aligned}
$$

where $\Psi(t)=\Psi_{c}(t)+\Psi_{m o d}(t)$ and where

- the expression (2) for $P h$ is used in the case of an edge;

- the expression (4) for $P h$ in the case of a bar.
To summarize, the VODKA principle mainly involves:

- a Gaussian-like angular sensitivity function for each photoreceptor, mimicking the angular sensitivity function of flies' photoreceptors [32];

a joint micro-scanning movement of the two photoreceptors, mimicking the retinal micro-movements observed in single ommatidia of the fly compound eye [20];

- a temporal derivative of the photoreceptor output signals, similar to the high pass filtering function occurring in the first order neuron of the fly's compound eye [37], [38].

The VODKA output signal results from the two photoreceptor signals being suitably processed to obtain the ratio between the difference and the sum of the differentiated photoreceptor signals.

$$
S_{V O D K A}=\frac{\left|P h_{1}^{\prime}(\Psi(t))\right|-\left|P h_{2}^{\prime}(\Psi(t))\right|}{\left|P h_{1}^{\prime}(\Psi(t))\right|+\left|P h_{2}^{\prime}(\Psi(t))\right|}
$$

where $\Psi(t)$ is the sum of the angular position $\Psi_{c}$ of the contrasting feature and the small vibration $\Psi_{\text {mod }}(t)$.

The derivative filter used here was a classical analog band-pass filter ( $H_{\text {band-pass }}$ in Fig.3), in which the high-pass filter section acts as the differentiator proper and the low-pass filter section reduces the high frequency noise and prevents the subsequent analog-to-digital conversion from undergoing any aliasing effects.

When the sensor is placed in front of an edge, developing the expression $S_{V O D K A}(6)$ yields a remarkably simplified expression, under the assumption that:

1 ) the amplitude of $\Psi_{\text {mod }}$ is very small (thus, $\Psi_{\text {mod }} \approx 0$ but $\Psi_{\text {mod }}^{\prime} \neq 0$ ); 


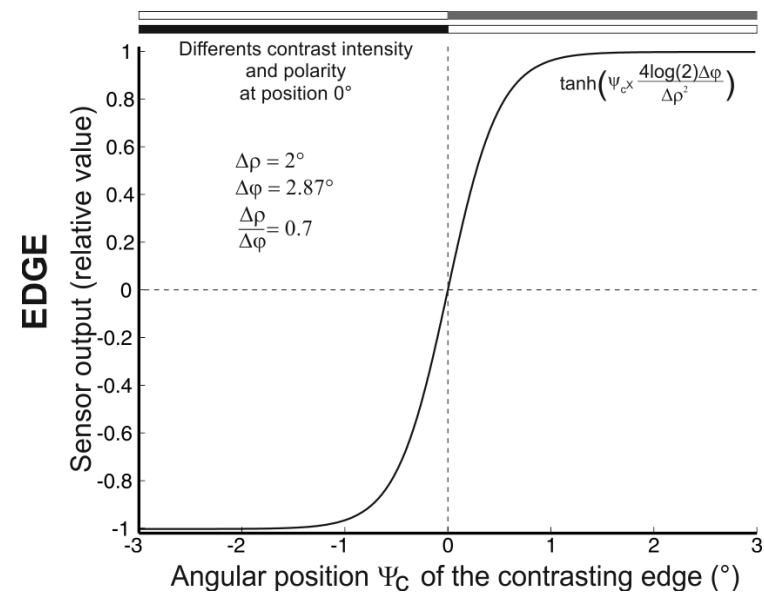

(a) Characteristic curve obtained in front of a contrasting edge

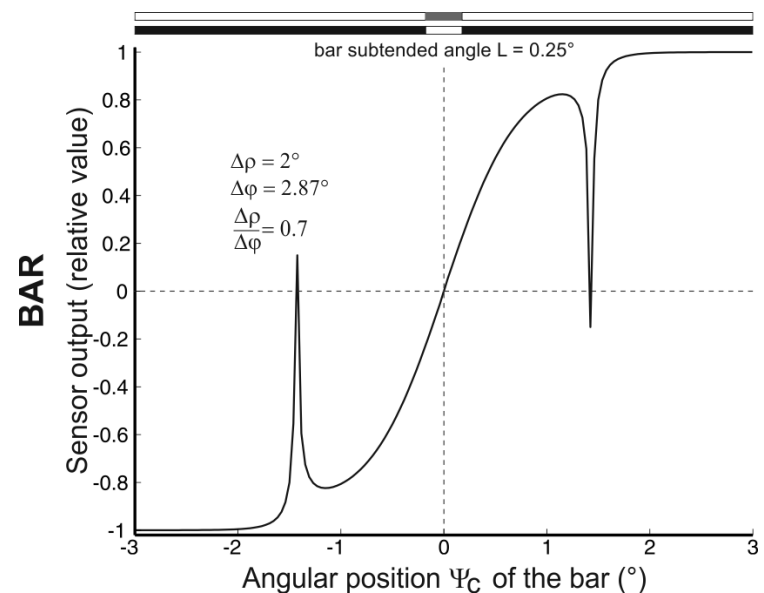

(b) Characteristic curve obtained in front of a contrasting bar (width $0.25^{\circ}$ )

Fig. 4. Theoretical characteristic curve of the VODKA sensor. When an edge is encountered (a), a tanh function is obtained (8). When a bar is encountered, there exists no obvious means of simplifying the expression of eq.(6) (here $\Delta \varphi=2.87^{\circ}$ and $\Delta \rho=2^{\circ}$ ).

2) the temporal variations of $\Psi_{c}$ are much slower than those of $\Psi_{\text {mod }}$ (thus, $\Psi_{c}^{\prime} \approx 0$ but $\Psi_{c} \neq 0$ ).

Under these conditions, the derivatives of the two photoreceptors can be expressed as follows, using eq.(1) and eq.(2):

$$
\begin{aligned}
& P h_{1}^{\prime}(\Psi(t))=k * \frac{\sqrt{\pi \ln (2)} \Psi^{\prime}(t)}{\pi \Delta \rho} * e^{-4 \ln (2) \frac{\left(\Psi(t)-\frac{\Delta \varphi}{2}\right)^{2}}{\Delta \rho^{2}}} \\
& P h_{2}^{\prime}(\Psi(t))=k * \frac{\sqrt{\pi \ln (2)} \Psi^{\prime}(t)}{\pi \Delta \rho} * e^{-4 \ln (2) \frac{\left(\Psi(t)+\frac{\Delta \varphi}{2}\right)^{2}}{\Delta \rho^{2}}}
\end{aligned}
$$

Substituting (7) into (6) gives, after some rewriting:

$$
S_{V O D K A}=\tanh \left(\Psi_{c} * \frac{4 \ln (2) \Delta \varphi}{\Delta \rho^{2}}\right)
$$

This remarkably simple expression gives the theoretical value of the VODKA sensor output as a function of the angular position $\Psi_{c}$ of an edge present in its FOV. The characteristic curve plotted in Fig.4a is this hyperbolic tangent function, which is an even, monotonic function of the angular position $\Psi_{c}$. The central part of the characteristic

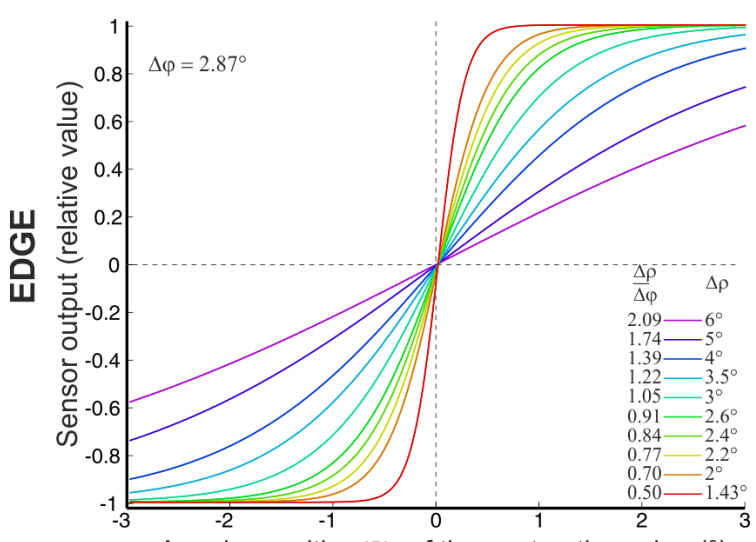

Angular position $\Psi_{\mathrm{C}}$ of the contrasting edge $\left({ }^{\circ}\right)$

(a) Theoretical characteristic curve for an edge

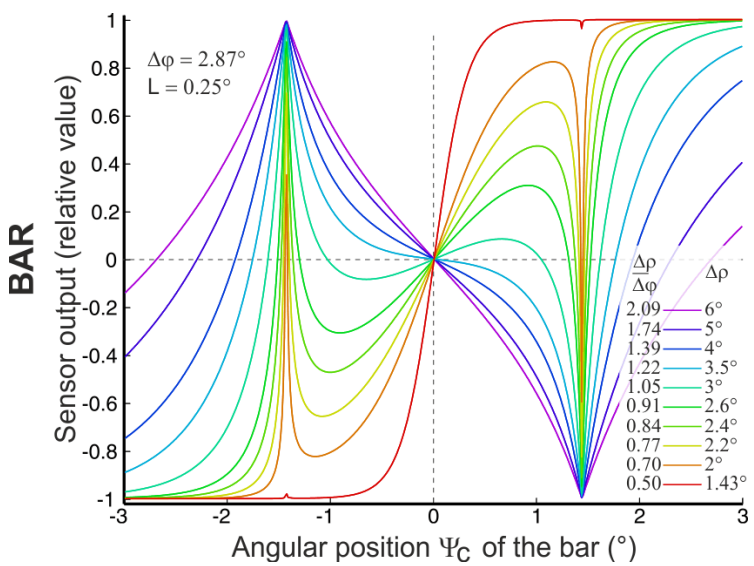

(b) $0.25^{\circ}$ Theoretical characteristic curve for a bar

Fig. 5. Effects of the optical parameters $\Delta \rho$ on the ability to locate an edge (a) and a bar (b)

curve varies quasi linearly with the angular position $\Psi_{c}$, which makes the sensor capable of locating the angular position of a contrasting edge with great accuracy.

When the sensor is placed in front of a contrasting bar, developing the expression $S_{V O D K A}$ [(6) with the temporal derivative of (4)] does not simplify easily. However, the expression obtained is still an even function, as shown in Fig.4b. It can be seen from this Figure that apart from the two "glitches " located at $( \pm \Delta \varphi / 2)$, the characteristic curve obtained in the case of a bar is fairly similar to that obtained in the case of an edge ( Fig.4 a and b).

The two parameters $\Delta \rho$ and $\Delta \varphi$ as well as the angular amplitude $A$ of the vibration and the width $L$ of the bar all affect the sensor's responses. The effects of these parameters are studied in Section IV.

\section{Parameters Affecting the Sensor's Output}

The two theoretical characteristic curves presented in Fig. 5 and in Fig. 6 were plotted from the equation (8) and [(6) with (2)] by taking the interreceptor angle $\Delta \varphi=2.87^{\circ}$, and the angle subtended by the bar to be $L=0.25^{\circ}$. These two Figures show the effects of a change in the ration $\Delta \varphi / \Delta \rho$, 


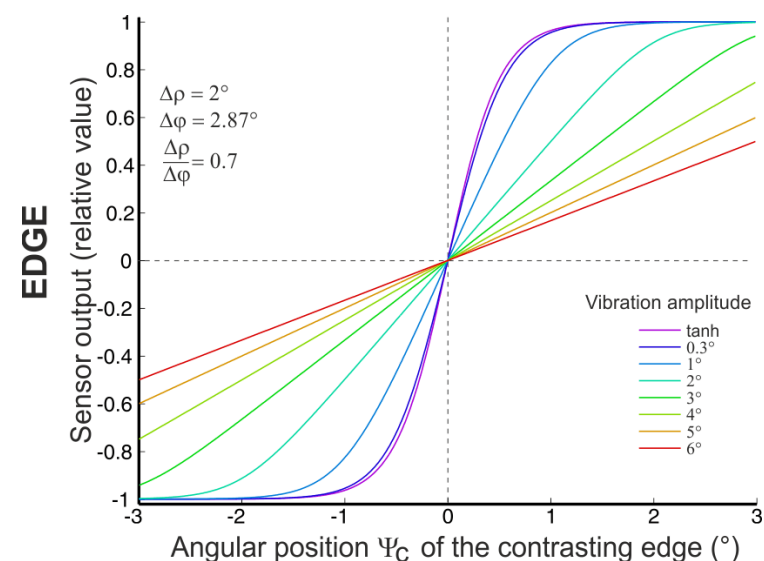

(a) Effects of the vibration amplitude $A$ on the theoretical characteristic curve in the case of an edge.

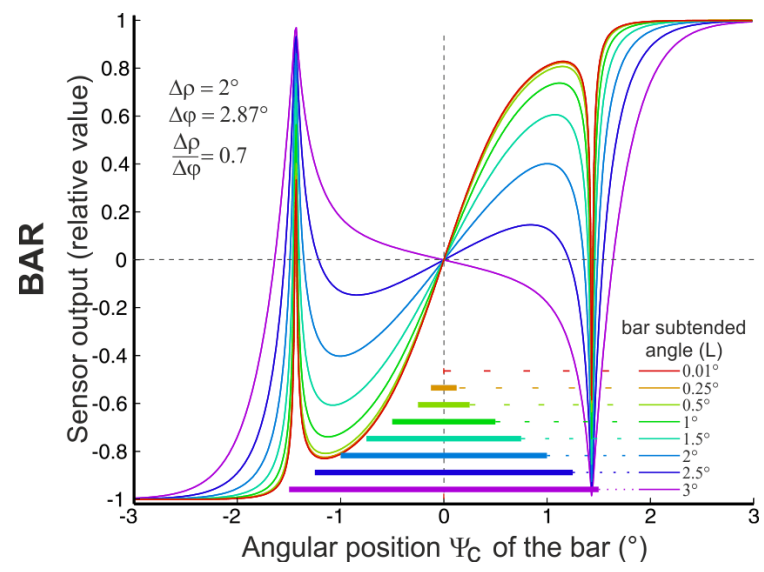

(b) Effects of the bar's angular width on the characteristic curve.

Fig. 6. Effects of various parameters on the theoretical characteristic curve.

a change in the scanning amplitude $A$, and a change in the angle subtended by the bar, respectively.

Fig. 5a presents the theoretical characteristic curve [obtained from (8)] of the VODKA sensor in the case of a contrasting edge when $\Delta \rho$ varies from $1.43^{\circ}$ to $6^{\circ}$. These values of $\Delta \rho$ correspond to a ratio $\Delta \rho / \Delta \varphi$ ranging from 0.5 to 2.09. This ratio is often used to characterize the optical properties of a compound eye [32]. Curves in Fig. 5a show that the lower the ratio $\Delta \rho / \Delta \varphi$, the more accurate the sensor becomes, at the expense of the sensor's angular range.

Fig. $5 \mathrm{~b}$ presents the theoretical characteristic curve obtained from (6) of the VODKA sensor in the case of a bar when the same parameter $\Delta \rho$ varies in the same range as in Fig.5a. Curves in Fig. 5b show that the sensor is capable of locating a bar only when the ratio $\Delta \rho / \Delta \varphi$ is smaller than 1 . When $\Delta \rho / \Delta \varphi$ is greater than 1 , the slope of the characteristic curve becomes negative in its central part. It is noteworthy that the characteristic curve for a bar is similar to that obtained for an edge when $\Delta \rho / \Delta \varphi=0.5$, showing that the sensor can locate a bar as well as an edge, provided the optical parameters are chosen appropriately.
Fig. 6a shows the effects of changes in the amplitude $A$ of the vibration on the theoretical characteristic curve (8) presented in Fig.4a. The theoretical result of equation (8) does not depend on the amplitude $A$. However, the effect of $A$ was determined by considering the averaging effect of the lowpass filter placed after the absolute value function (see Fig.3). Therefore, in the case of a triangular (linear) periodic scanning law applied to the retina, the average over one temporal period (noted $T_{\Psi}$ ) is:

$$
\begin{aligned}
\frac{P h_{1}^{\prime}-P h_{2}^{\prime}}{P h_{1}^{\prime}+P h_{2}^{\prime}} & =\int_{0}^{T_{\Psi}} \frac{1}{T_{\Psi}} \tanh \left(\Psi(t) * \frac{4 \ln (2) \Delta \varphi}{\Delta \rho^{2}}\right) d t \\
& =\frac{1}{2 A} \int_{-A}^{A} \tanh \left(\left(\Psi_{c}(t)+x\right) * \frac{4 \ln (2) \Delta \varphi}{\Delta \rho^{2}}\right) d x
\end{aligned}
$$

After developing and simplifying these expressions, (9) becomes:

$$
\frac{\Delta \rho^{2}}{2 A * 4 \ln (2) \Delta \varphi} *\left(\ln \frac{\cosh \left(\frac{4 \ln (2) \Delta \varphi}{\Delta \rho^{2}} *\left(\Psi_{c}(t)+A\right)\right)}{\cosh \left(\frac{4 \ln (2) \Delta \varphi}{\Delta \rho^{2}} *\left(\Psi_{c}(t)-A\right)\right)}\right)
$$

Equation (10), plotted in Fig. 6a, shows the influence of the scanning amplitude $A$ on the characteristic curve of the VODKA sensor. It is worth noting that when the scanning amplitude $A$ tends to be small or null, equation (10) tends to be equal to equation (8). Fig. 6a shows that in the case of a very small amplitude $A\left(A=0.3^{\circ}\right.$, which is about 10 times smaller than the interreceptor angle $\Delta \varphi$ ) the theoretical characteristic curve nearly matches the theoretical tanh (8) curve.

Fig. $6 \mathrm{~b}$ shows the effect of a change in the angle $L$ subtended by the bar on the predicted characteristic curve obtained from equations (6) and (4). Interestingly, the change in $L$ has similar effects on the linearity of the characteristic curve to those observed in Fig. 5b, where only the ratio $\Delta \rho / \Delta \varphi$ varied.

To summarize, several parameters liable to affect the characteristics of the sensor output were modeled and characterized for a contrasting edge (see Fig. 5a and 6a) and for a bar (see Fig. $5 b$ and $6 b$ ). Therefore, to obtain a suitable characteristic curve, both the ratio $\Delta \rho / \Delta \varphi$ and the scanning amplitude $A$ can be selected and set accurately. The effect of a few other parameters was studied in [8].

\section{Noteworthy Features of the VODKA Sensor}

\section{A. Robustness to Lighting Conditions}

The temporal derivation, which is part of the frontend bandpass filter (see Fig. 3) amplifies high frequencies and removes the DC component from each photoreceptor output signal. Very large AC signal gain amplification can be obtained since the DC value is filtered out. These filtering and amplification steps in the signal processing make the VODKA sensor relatively robust to the lighting conditions (affecting the DC value) at this early stage in the signal processing. 


\section{B. Robustness to Contrast Value and Contrast Polarity}

It is the absolute value of signals $P h_{1}^{\prime}$ and $P h_{2}^{\prime}$ that is used in equation (6). The sensor is therefore robust to the polarity of the contrasting feature encountered (an edge or a bar), that is, it will respond equally to a white-to-dark or a dark-to-white target.

By calculating the relative difference [with expression (6)], the influence of contrast [modeled by the factor $k$ in equations (2) and (4)] can be removed. However, this is a theoretical result, and the experimental data obtained with the sensor we implemented show a minimum detectable contrast (11) of about $5 \%$.

\section{Robustness to Vibration Types}

Joint vibration of the two optical axes affects the signals delivered by both photoreceptors. From the amplitude of these signals, the angular position $\Psi_{c}$ of a contrasting target lying in VODKA's visual field is obtained. From equation (8), it can be seen that the vibration frequency has no influence on the ability of the sensor to locate the target. Consequently, not only periodic signals such as sine waves but also random patterns of vibration can be used. For example, the "natural" undesirable vibration produced by the sensor's mechanical support (e.g., a robotic platform) can be exploited to drive the VODKA sensor's visual processing system, as described in part VI-B.

According to the VODKA principle described in section III, a vibration pattern of some kind is a prerequisite to obtain hyperacuity, even though the type of vibration has no effect on the predicted sensor output. Taking the case of the low pass filter in the digital demodulation scheme (see Fig. 3), for example, the best results will be obtained with relatively high frequency and low amplitude vibrations [because the derivative of the high frequency in (7) counteracts the low vibration amplitude].

\section{EXPERIMENTAL RESULTS}

In the first experiment (Fig.8), the sensor with its vibrating retina was kept stationary in front of a contrasting edge that was set into slow translation (see Fig.7). In the second experiment (Fig.9), the VODKA sensor was mounted onboard the 100g OSCAR 2 aerial robot [9], which is able to rotate freely in yaw. Here, the natural vibrations produced by the robot itself (vibrations generated by the two propellers) were exploited by the VODKA sensor and used to control the robot's yaw orientation. Illuminance was varied (from 118 Lux to 5 Lux) to test the robustness of the sensor to dimly lit environments.

In these two experiments, the digital filters $H_{\text {peak }}$ and $H_{\text {low-pass }}$ (Fig.3) were suitably tuned to meet the requirements of the experimental situation: the VODKA principle can be adapted to work in various situations and to achieve various objectives.

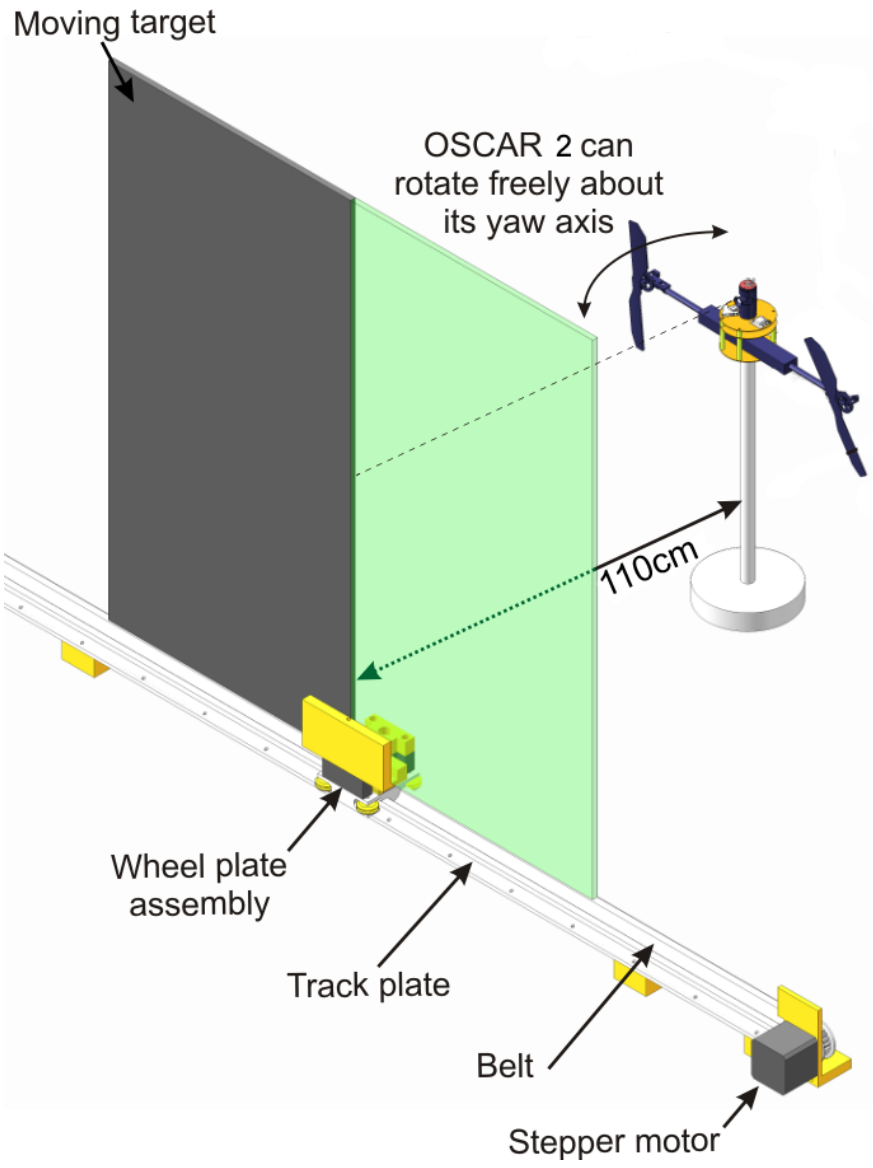

Fig. 7. CAD of the experimental setup. The aerial robot (OSCAR 2, see Fig.1) is placed $1.1 \mathrm{~m}$ ahead of a vertical edge that is set in horizontal motion in micro-steps of $62 \mu \mathrm{m}$. In the first experiment (section VI-A), the robot's propellers were switched off (i.e., the robot was stationary) and the VODKA sensor's retina was vibrated at a high frequency $\left(f_{a}=40 \mathrm{~Hz}\right)$ by means of the piezo-bender (see Fig.2 and 3). The output of the VODKA sensor was monitored via a BlueTooth connection, while the contrasting edge was slowly translated (hence modifying the angle $\Psi_{c}$ ). In the second experiment (section VI-B), the robot's propellers were switched on and the piezo driver was switched off. The VODKA sensor used the robot's natural vibrations. Mounted on the axis of a frictionless resolver, the robot was free to rotate about its yaw axis and tracked the contrasting edge accurately on the basis of what it saw. (adapted from [9].

Fig. 7 shows the test bench used in the two experiments. The contrasting edge used here had a high contrast: $m=0.85$, based on the Michelson contrast definition:

$$
m=\frac{I_{1}-I_{2}}{I_{1}+I_{2}}
$$

where the light intensities $I_{1}$ and $I_{2}$ are the higher and lower luminances measured on the two surfaces, respectivly ( $I_{1}$ and $I_{2}$ measured with a similar photoreceptor to the VODKA photoreceptors).

The digital fixed point processing of the VODKA sensor was implemented in a 16-bit microcontroller (dsPIC30f4013) running at 20 MIPS. It requires only a very small part (3.3\%) of the available calculation resources. Program code was generated with a simulink Real time Workshop embedded target and the custom made dsPIC blockset [39]. 


\section{A. VODKA: a Hyperacute Visual Sensor}

Fig. 8 shows the sensor's output when a contrasting edge (contrast $m=0.85$ ) placed 1.1 meter ahead was slowly moved along the bench.

In this experiment, the robot was stationary and the two optical axes were vibrated in concert via the piezo-bender set in the eye tube, as depicted in Fig.2. The input signal to the piezo-bender was a sine wave with a frequency of $40 \mathrm{~Hz}$ and an amplitude $A=0.2^{\circ}$ (creating thus the angular vibration noted $\Psi_{m o d}$ ). Each translational micro-step displacement of the target was equivalent to a rotational microstep of $0.0032^{\circ}$ in the angular position $\Psi_{c}$ of the edge. As predicted in section III [(6) and Fig.4a, Fig.5a, Fig.6a], the experimental characteristic curve in Fig. 8 was a hyperbolic tangent function of the angular position $\Psi_{c}$. The inset in Fig. 8 illustrates the remarkable property of hypercuity with which the VODKA sensor is endowed, since each micro step displacement $\left(0.0032^{\circ}\right)$ led to a noticeable change in the response of the sensor as detailed below. Since the static resolution of the sensor is determined by the interreceptor angle $\left(\Delta \varphi=2.87^{\circ}\right)$, the retinal micro-scanning process can be said to enhance the resolution in the task of locating an edge by an outstandingly high factor of $2.87 / 0.0032=897$.

Let us compute a few statistics on the 21 consecutive sensor output values (corresponding to the 21 dots traced at each target position on Fig. 8, and in the inset of Fig. $8)$. The target displacement from the angular position $-1^{\circ}$ to $+1^{\circ}$ is done through 615 micro-step offsets. Within theses 615 positions, the mean of the intra-step standard deviation is $\sigma_{\text {Svodka }}=0.922 * 10^{-3}$ and the mean of the interquartile range is $I Q R_{\text {Svodka }}=0.817 * 10^{-3}$. The sensor's output $S_{\text {vodka }}$ increases quasi linearly from -0.8 at $-1^{\circ}$ to 0.8 at $1^{\circ}$. Consequently, the mean inter-step distance is $\Delta_{\text {Svodka }}=2.6 * 10^{-3}$ that is about three times the standard deviation of intra-step measurement. Thus, each of theses 615 angular positions can be resolved from the VODKA sensor output.

To achieve such a highly accurate target angular detection performance, the various filters involved in the visual processing system (see Fig. 3) were tuned with the following parameters:

The transfer function $H_{\text {band-pass }}$ of the analog band-pass filter (detailed in [8]) is given by:

$$
H_{\text {band-pass }}=30 * \frac{50 * 2 \pi}{s+50 * 2 \pi} * \frac{s}{s+50 * 2 \pi}
$$

The transfer function $H_{P e a k}$ of the digital peak filter (peak frequency $f_{a}=40 \mathrm{~Hz}$, sampling frequency $500 \mathrm{~Hz}$ ) is given by:

$$
H_{\text {Peak }}=0.036 * \frac{z^{2}-1}{z^{2}-1.68 z+0.93}
$$

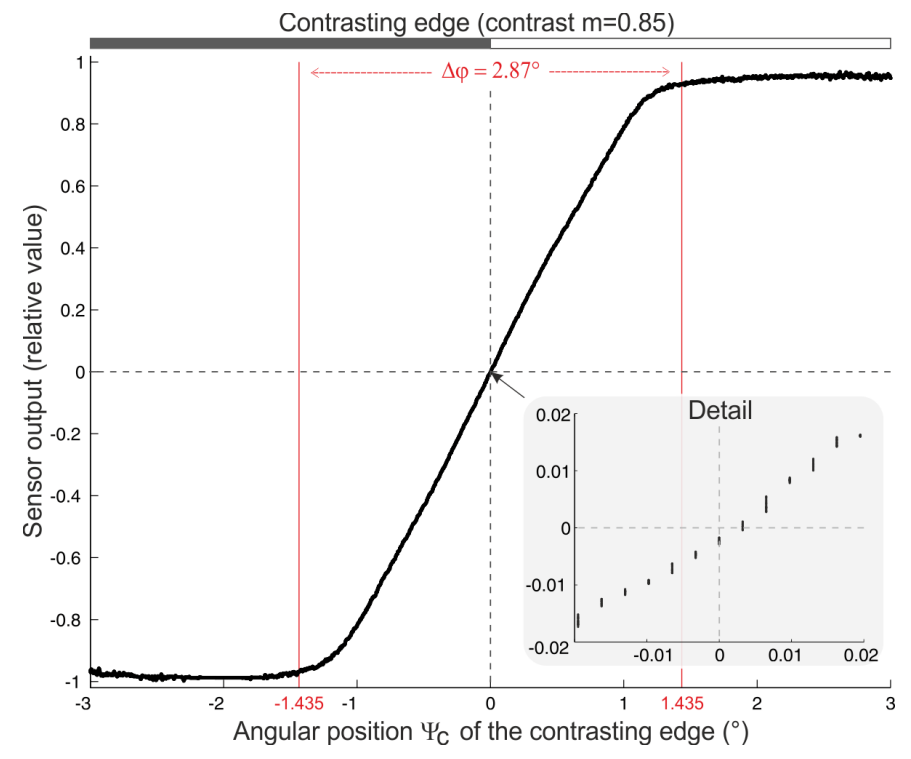

Fig. 8. Experimentally determined characteristic curve of the implemented VODKA sensor. Relative sensor output as a function of the angular position $\Psi_{c}$ of an edge (contrast $m=0.85$ ) placed $1.1 m$ ahead, at an illuminance of approximatly 120 lux. The edge was displaced slowly via a stepper motor in micro steps of $62 \mu \mathrm{m}$, corresponding to an angular shift of only $0.0032^{\circ}$ (Fig. 7). In this Figure, 21 consecutive sensor outputs were measured and plotted for each step position. The experimental curves fit the theoretical hyperbolic tangent function of the contrast position $\Psi_{c}$ [(8), Fig.4a]. The thinness of the resulting curve shows the high resolution obtained. The lower right inset gives a highly magnified picture of the central part of the characteristic curve within an angular range of only $\pm 0.02^{\circ}$. The parameters used here were $\Delta \varphi=2.87^{\circ}, \Delta \rho=3^{\circ}$, scan shape: sine wave, scan frequency: $f_{a}=40 \mathrm{~Hz}$, scan amplitude: $A=0.2^{\circ}$.

Lastly, the transfer function of the digital second order low-pass filter $H_{\text {low-pass }}$ (cut-off frequency $5 \mathrm{~Hz}$, sampling frequency $500 \mathrm{~Hz}$ ) is given by:

$$
H_{\text {low-pass }}=9.447 * 10^{-4} \frac{(z+1)^{2}}{z^{2}-1.911 z+0.915}
$$

\section{B. Pursuit of a Moving Target Using the Robot's Own Vibra- tions}

The results presented in Fig. 9 show the tracking performance of which the VODKA sensor is capable, using only the vibrations naturally generated by its own two propellers. The frequencies of these vibrations are visible on the rate gyro spectrogram (curve d), and on the spectrogram obtained with one photoreceptor derivative (curve c). The piezo-bender shown in Fig.2 was not activated here and the retina therefore remained stationary behind its lens. This experiment first confirms the accuracy of the sensor and its robustness to a relatively wide range of lighting conditions (curve a), from DC lighting (switched to various illuminances) to fluorescent lighting [which causes strong $100-\mathrm{Hz}$ interferences in each photoreceptor signal (as attested by curve b, from $97 \mathrm{~s}$ to $120 \mathrm{~s}$ )].

The robot's yaw angular orientation was monitored accurately by the miniature resolver endowed with neglegible friction (Fig.1). The robot's yaw control depended only on the output signal yielded by the embedded VODKA sensor. The 


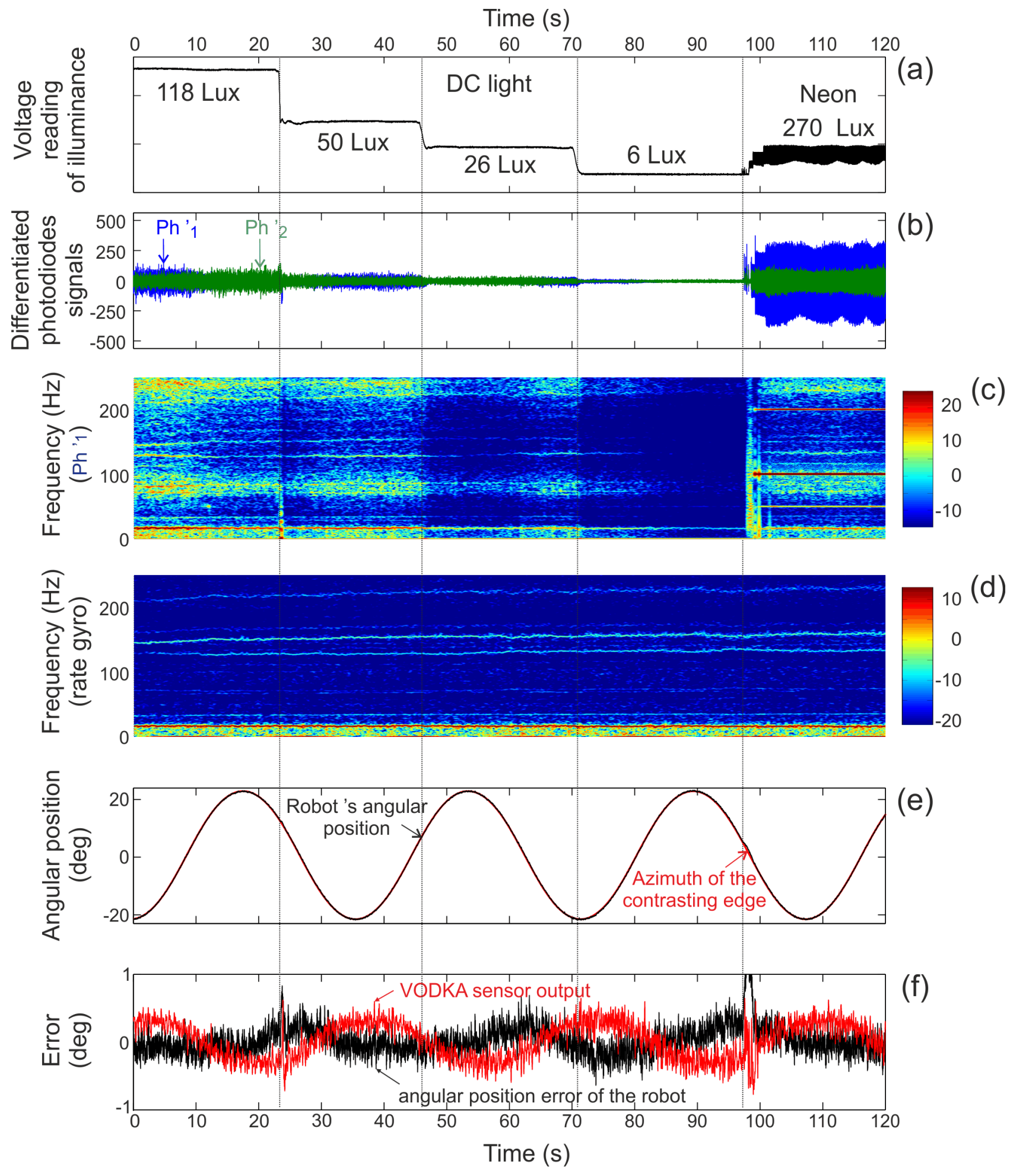

Fig. 9. Smooth pursuit of a contrasting edge (contrast $m=0.85$ ) set in sinusoidal motion in front of the tethered OSCAR 2 robot (Fig. $1 \& 7$ ) equipped with the VODKA sensor (see Fig.2 \& 3). Here, the piezo-bender (on Fig.2) is inactive and the VODKA sensor makes use of the natural random vibrations caused by the two robot propellers. Curve (a) shows that the DC light intensity was decreased about twenty-fold (from 118 Lux to 6 Lux) and finally switched to Neon room lighting (after 97s). Curve (b) shows the two photoreceptors' derivatives $P h_{1}^{\prime}$ and $P h_{2}^{\prime}$, and curve (c) shows the spectrogram of $P h_{1}^{\prime}$. Curve (d) shows the spectrogram of an embedded rate gyro measuring the yaw rate. It can be seen from the spectrograms in (c) and (d) that the robot's vibrations affect the photoreceptor signal at the typical frequencies $(16 \mathrm{~Hz}, 125 \mathrm{~Hz}, 150 \mathrm{~Hz}$ and $210 \mathrm{~Hz}$ ) observable in the robot's own yaw spectrogram (d). The curves in (e) show the angular position of the translating edge (in red), and the angular orientation of the OSCAR robot (in black), respectively. At all times, the robot realigns itself towards the edge accurately, based on the VODKA sensor output. These two curves are remarkably well superimposed despite the illuminance changes. Curves (f) show (in a magnified form) the angular tracking error (in black), which is the difference between the two curves in (e), and the VODKA visual sensor's output (in red), which controls the robot's yaw orientation. This experiment confirms the ability of theVODKA sensor to make use of natural vibrations and shows that it is relatively robust to the lighting conditions. The VODKA output signal itself (f, red curve) is hardly affected at all by the dimming of the DC light or by the presence of artificial light, although the latter causes major interferences in the photoreceptors' derivatives [curve (b), from $97 \mathrm{~s}$ to $120 \mathrm{~s}]$. 
rotational speed of each propeller was driven differentially by a feedback control loop based on the VODKA sensor's output signal (f:red curve) and involving some state-space controler detailed in [9].

The edge was moved sinusoidally in front of the robot [the black curve in (e) shows the azimuthal orientation of the target]. The robot can be seen to have followed the target faithfully (e). The standard deviation of the robot's orientation error signal (f:black curve) turned out to be only $\sigma=0.15^{\circ}$. This value [approximately 20 -fold smaller than the static resolution of the robot's eye $\left(2.87^{\circ}\right)$ ] is very small, especially if one takes into account the additional error introduced by the control process of the aerial robot itself (the $100 \mathrm{~g}$ robot attained its steady yaw rate with a time constant of $306 \mathrm{~ms}$ in response to a differential open loop step command over its two propellers). The result of this experiment therefore confirms that the VODKA principle can be implemented to meet various requirements in terms of accuracy and dynamics as a means of controlling an aerial robot effectively and safely.

The filters present in the visual processing scheme described in Fig. 3 were adapted to be able to meet the dynamic and precision requirements using random vibrations.

The transfer function $H_{\text {band-pass }}$ was not changed and is therefore given by equation (12).

The transfer function $H_{P e a k}$ in equation (13) is not appropriate because the visual signal is not modulated periodically at $40 \mathrm{~Hz}$. The $H_{P e a k}$ filter was therefore replaced by a comb filter of order 10 with a quality factor $Q=10$ running at $500 \mathrm{~Hz}$ that removes both the DC part of the signal and the $50 \mathrm{~Hz}$ with all its harmonics created by the neon lighting. The $H_{C o m b}$ transfer function replaces the $H_{P e a k}$ transfer function:

$$
H_{C o m b}=0.7729 * \frac{z^{10}-1}{z^{10}-0.5459}
$$

The remaining signals are then mostly due to the vibration of the optical axes.

The cut-off frequency of the transfer function $H_{\text {low-pass }}$ was increased to $10 \mathrm{~Hz}$ so as to increase the dynamic response of the sensor. The new transfer function (sampling frequency of $500 \mathrm{~Hz}$ ) is:

$$
H_{\text {low-pass }}=3.622 * 10^{-3} * \frac{(z+1)^{2}}{z^{2}-1.823 z+0.8372}
$$

\section{CONCLUSION}

The VODKA sensor is a highly accurate optical Position Sensing Device (PSD). VODKA is able to determine the angular position of a naturally lit target by extracting and processing the low amplitude and high frequency signals transmitted by two jointly vibrating photoreceptors. Even in its elementary form described here (only two pixels), the VODKA sensor was found to be capable of locating the angular position of an edge with a far better (900-fold better) resolution than that anticipated from the static resolution (as determined on the basis of the interreceptor angle $\Delta \varphi$ ) regardless of the scanning law and scanning frequenc. The tremendous hyperacuity factor achieved by this two-pixelretina results from (i) the micro-vibration of the retina behind the lens and (ii) the specific signal processing of the visual signals. The evenness of the characteristic curve obtained (Fig. 8) shows that the sensor is a good candidate for being integrated into visuomotor control loops - in the context of micro aerial vehicle stabilization and tracking, for example. In another biomimetic approach, Lichtsteiner et al. built a CMOS vision sensor [40] encoding the pixel intensity changes. This asynchronous CMOS imager was incorporated into an original demonstrator, in terms of a fast visual feedback control loop that was able to balance a pencil [41] with a latency smaller than $2 \mathrm{~ms}$. Despite this small latency, the accuracy of the visual servo-loop was strongly dependent on the imager resolution (128x128 pixels). In future developments of the VODKA sensor, an increase in the scanning frequency should likely overcome the trade-off existing between accuracy and refresh rate. In any case, the hyperacuity achieved by the VODKA sensor in locating an edge does not rely on using an array or a matrix of photoreceptors, and it is obtained already when using just two photodiodes.

All in all, the VODKA sensor is an Position Sensing Device (PSD) featuring the following remarkable properties:

1) It is able to locate a contrasting edge with an outstanding precision (ca 900-fold hyperacuity factor).

2) It responds to naturally lit objects.

3) It is insensitive to contrast polarity ( $\mathrm{ON}$ edges or OFF edges).

4) It is largely insensitive to contrast intensity (down to about $5 \%$ [8]).

5) It responds in the presence of interferences (e.g. $100 \mathrm{~Hz}$ or $120 \mathrm{~Hz}$ caused by artificial lighting: Fig. 9).

6) It responds within a relatively large range of illuminance (factor 20: Fig. 9) despite its nonadaptive photoreceptors.

7) It features a low response latency (35ms at the vibration frequency tested, $f_{a}=40 \mathrm{~Hz}$ [8]).

8) It is low weight, powerlean and simple to implement.

9) It can be organized in arrays of similar sensors to build an imager with outstanding positioning accuracy within a large FOV [42].

10) The parallel architecture of such a sensor array is appropriate for analog VLSI implementation, all the more since the elementary processing requires only nextneighbor interactions.

The basic principles described here are still applicable in the case of random vibrations of the eye, such as vibrations produced by the propellers of an aircraft. We have shown an example of the highly accurate heading and tracking ability of a miniature aerial robot equiped with a VODKA sensor exploiting the uncontrolled, random vibrations generated by the propellers. This unique property makes the sensor much simpler to construct since it can do without any embedded microscanners. Although the VODKA principle can cope with 
natural vibrations, it remains that imposing specific vibrations upon the retina by means of a dedicated micro-actuator (piezobender, electromagnetic actuators, etc...) yields better performance because the signal processing steps can be finely tuned to the (known) scan frequency, allowing the modulated visual output signals to be extracted with a better signal-to-noise ratio. Since the VODKA sensor shares the same processing front end (the band-pass filter) as our optic flow sensors [43] [46], it is possible to obtain dual optical position and optic flow sensors sharing the same FOV, and the same (optical and electronical) frontend hardware. [8], [42], [47].

On the one hand, the simplicity, parallel architecture and small size of the VODKA hyperacute position sensing device make it appropriate for applications in the fields of metrology, robotics, astronomy, automotive and aerospace engineering. All the more so than one may extend the VODKA principle to a large imager, whose resolution will be enhanced across its whole FOV without the cost of large memory resources [42]. On the other hand, the hyperacuity achieved in the VODKA sensor may shed new light on the principles underlying the miniature eye movements (tremor) that have been described in both vertebrates' camera eyes and arthropods' compound eyes.

\section{ACKNOWLEDGMENT}

The authors acknowledge the assistance of M. Boyron in designing and producing the miniature electronic boards used here, including the piezo driver. They also thank Y. Luparini for his help with the mechanical construction of the robot. We thank J. Blanc for revising the English manuscript. This work was supported by the CNRS, the University of the Mediterranean, the French National Research Agency (ANR with the RETINAE and EVA projects) and the European Union (Future and Emerging Technologies, Seventh Framework Programme for Research of the European Commission, FET-Open grant number: 237940 (CURVACE project).

\section{REFERENCES}

[1] G. Westheimer, Visual hyperacuity. Berlin: Ottoson, Sensory Physiology 1, Springer, 1981.

[2] S. Martinez-Conde, S. .Macknik, and D. H. Hubel, "The role of fixational eye movements in visual perception." Nat Rev Neurosci, vol. 5, no. 3, pp. 229-240, Mar 2004. [Online]. Available: http://dx.doi.org/10.1038/nrn1348

[3] H.-K. Ko, M. Poletti, and M. Rucci, "Microsaccades precisely relocate gaze in a high visual acuity task." Nat Neurosci, vol. 13, no. 12, pp. 1549-1553, Dec 2010. [Online]. Available: http: //dx.doi.org/10.1038/nn.2663

[4] M. H. Hennig and F. Wörgötter, "Eye micro-movements improve stimulus detection beyond the nyquist limit in the peripheral retina," in NIPS, vol. 16. MIT press, 2004.

[5] S. C. Chen, L. E. Hallum, G. J. Suaning, and N. H. Lovell, "Psychophysics of prosthetic vision: I. visual scanning and visual acuity," in Proc. 28th Annual International Conference of the IEEE Engineering in Medicine and Biology Society EMBS '06, Aug. 30 2006-Sept. 3 2006, pp. $4400-4403$.

[6] R. A. Altes, "Ubiquity of hyperacuity," The Journal of the Acoustical Society of America, vol. 85, no. 2, pp. 943-952, 1989. [Online]. Available: http://link.aip.org/link/?JAS/85/943/1

[7] L. Kerhuel, S. Viollet, and N. Franceschini, "A sighted aerial robot with fast gaze and heading stabilization," in Proc. of IEEE Conf on Intelligent Robots and Systems (IROS), San Diego, CA, USA, October 2007, pp. 2634-2641.
[8] L. Kerhuel, "Capteurs optiques minimalistes et réflexes oculomoteurs biomimétiques ; application à la robotique aérienne," Ph.D. dissertation, Université de Montpellier II, December 2009. [Online]. Available: http://tel.archives-ouvertes.fr/tel-00559101

[9] L. Kerhuel, S. Viollet, and N. Franceschini, "Steering by gazing: An efficient biomimetic control strategy for visually guided micro aerial vehicles," IEEE Transactions on Robotics, vol. 26, no. 2, pp. 307-319, 2010.

[10] E. P. Krotkov, "Visual hyperacuity: Representation and computation of high precision position information," Computer Vision, Graphics, and Image Processing, vol. 33, no. 1, pp. 99 - 115, 1986. [Online]. Available: http://www.sciencedirect.com/science/article/ B7GXG-4CTNF6S-5/2/e13db0d140eed2b50d5e86c58a7efb29

[11] D. T. Riley, W. M. Harmann, S. F. Barrett, and C. H. G. Wright, "Musca domestica inspired machine vision sensor with hyperacuity," Bioinspiration \& Biomimetics, vol. 3, no. 2, June 2008. [Online]. Available: http://stacks.iop.org/1748-3190/3/026003

[12] A. Brückner, J. Duparré, A. Bräuer, and A. Tünnermann, "Artificial compound eye applying hyperacuity," Opt. Express, vol. 14, no. 25, pp. 12 076-12 084, 2006. [Online]. Available: http://www.opticsexpress. org/abstract.cfm?URI=oe-14-25-12076

[13] S. Morokawa, "Image pickup system," US Patent US4 581 649, 1986.

[14] P. Seitz, "Optical superresolution using solid-state cameras and digital signal processing." Optical Engineering, vol. 27, pp. 535-540, 1988.

[15] P. Chevrette and J. Fortin, "Method and system for fast microscanning," USA Patent US5 774 149, 1998.

[16] A. M. Koekemoer et al., Hubble Space Telescope dither handbook, 2nd ed. Baltimore: STScI, 2002. [Online] Available: http://www.stsci.edu/hst/HST_overview/documents/dither_ handbook/dither_handbook_v2.pdf

[17] M. F. Land, "Movements of the retinae of jumping spiders (salticidae: dendryphantinae) in response to visual stimuli." J Exp Biol, vol. 51, no. 2, pp. 471-493, Nov 1969.

[18] Kaps and Schmid, "Mechanism and possible behavioural relevance of retinal movements in the ctenid spider cupiennius salei," J Exp Biol, vol. 199, no. Pt 11, pp. 2451-2458, 1996.

[19] M. Land, "Scanning eye movements in a heteropod mollusc," $J$ Exp Biol, vol. 96, no. 1, pp. 427-430, Feb. 1982. [Online]. Available: http://jeb.biologists.org

[20] N. Franceschini and R. Chagneux, "Repetitive scanning in the fly compound eye," in Göttingen Neurobiology Report, vol. 2. Thieme, 1997, p. 279.

[21] F. Mura and N. Franceschini, "Obstacle avoidance in a terrestrial mobile robot provided with a scanning retina," in Proc. IEEE Intelligent Vehicles Symposium, 19-20 Sept. 1996, pp. 47-52.

[22] S. Viollet and N. Franceschini, "Biologically-inspired visual scanning sensor for stabilization and tracking," in Intelligent Robots and Systems, 1999. IROS '99. Proceedings. 1999 IEEE/RSJ International Conference on, vol. 1, 17-21 Oct. 1999, pp. 204-209.

[23] K. Hoshino, F. Mura, and I. Shimoyama, "A one-chip scanning retina with an integrated micromechanical scanning actuator," IEEE J. MEMS, vol. 10 , no. 4, pp. 492-497, Dec. 2001.

[24] S. Viollet and N. Franceschini, "A hyperacute optical position sensor based on biomimetic retinal micro-scanning," Sensors and Actuators A: Physical, vol. 160, no. 1-2, pp. 60 - 68, 2010. [Online]. Available: http://www.sciencedirect.com/science/article/ B6THG-4YR8RCS-3/2/3c2332af09653720f27c59080539e747

[25] F. Mura and I. Shimoyama, "Visual guidance of a small mobile robot using active, biologically-inspired, eye movements," in Proc. IEEE International Conference on Robotics and Automation, vol. 3, 16-20 May 1998, pp. 1859-1864.

[26] S. Viollet and N. Franceschini, "Visual servo system based on a biologically inspired scanning sensor," in Sensor Fusion and Decentralized control in Robotics II, vol. 3839. SPIE, 1999, pp. 144-155.

[27] S. Viollet and N. Franceschini, "A high speed gaze control system based on the vestibulo-ocular reflex," Robotics and Autonomous systems, vol. 50, pp. 147-161, 2005 .

[28] O. Landolt and A. Mitros, "Visual sensor with resolution enhancement by mechanical vibrations," Autonomous Robots, vol. 11, no. 3, pp. 233-239, Nov. 2001. [Online]. Available: http://dx.doi.org/10.1023/A: 1012482822516

[29] P. N. Prokopowicz and P. R. Cooper, "The dynamic retina: Contrast and motion detection for active vision," International Journal of Computer Vision, vol. 16, pp. 191-204, 1995, 10.1007/BF01539626. [Online]. Available: http://dx.doi.org/10.1007/BF01539626

[30] M. O. Hongler, Y. L. de Meneses, A. Beyeler, and J. Jacot, "The resonant retina: exploiting vibration noise to optimally detect edges in an 
image," Pattern Analysis and Machine Intelligence, IEEE Transactions on, vol. 25, no. 9, pp. 1051-1062, Sept. 2003.

[31] S. Viollet, F. Ruffier, T. Ray, M. Menouni, F. Aubépart, L. Kerhuel, and N. Franceschini, "Characteristics of three miniature bio-inspired optic flow sensors in natural environments," in Proc. Fourth Int Sensor Technologies and Applications (SENSORCOMM) Conf, 2010, pp. 5155.

[32] K. G. Götz, "Optomotorische Untersuchengen des visuellen Systems einiger Augnmuntanten der Fruchfliege Drosophila," Kybernetik, vol. 2, pp. 77-92, 1964.

[33] N. Franceschini and K. Kirschfeld, "Etude optique in vivo des éléments photorécepteurs dans l'oeil composé de drosophila," Biological Cybernetics, vol. 8, no. 1, pp. 1-13, Jan. 1971. [Online]. Available: http://dx.doi.org/10.1007/BF00270828

[34] A. W. Snyder, Handbook of Sensory Physiology. Springer-Verlag Berlin Heidelberg New York, 1979, vol. VII/6A, ch. Physics of Vision in Compound Eyes, pp. 225-313.

[35] D. G. Stavenga, "Angular and spectral sensitivity of fly photoreceptors. i. integrated facet lens and rhabdomere optics." J. Comp. Physiol. $A$, vol. 189, no. 1, pp. 1-17, Jan 2003. [Online]. Available: http://dx.doi.org/10.1007/s00359-002-0370-2

[36] D. G. Stavenga, "Angular and spectral sensitivity of fly photoreceptors. ii. dependence on facet lens f-number and rhabdomere type in drosophila." J. Comp. Physiol. A, vol. 189, no. 3, pp. 189-202, Mar 2003. [Online]. Available: http://dx.doi.org/10.1007/s00359-003-0390-6

[37] F. Zettler and R. Weiler, Neural principles in vision. Berlin: Springer, 1974 , ch. Neuronal processing in the first optic neuropile of the compound eye of the fly, pp. 226-237.

[38] M. Juusola and A. S. French, "Visual acuity for moving objects in firstand second-order neurons of the fly compound eye." J Neurophysiol, vol. 77, no. 3, pp. 1487-1495, Mar 1997.

[39] L. Kerhuel, "dspic blockset for simulink," October 2008. [Online]. Available: http://www.kerhuel.eu

[40] P. Lichtsteiner, C. Posch, and T. Delbrück, "A 128 x 128 120db 30mw asynchronous vision sensor that responds to relative intensity change," in Proc. Digest of Technical Papers. IEEE Int. Solid-State Circuits Conf. ISSCC 2006, 2006, pp. 2060-2069.

[41] J. Conradt, M. Cook, R. Berner, P. Lichtsteiner, R. J. Douglas, and T. Delbrück, "A pencil balancing robot using a pair of AER dynamic vision sensors," in Proc. IEEE Int. Symp. Circuits and Systems ISCAS 2009, 2009, pp. 781-784.

[42] L. Kerhuel, N. Franceschini, and S. Viollet, "Method and device for measuring the angular position of a rectilinear contrasting edge of an object, and system for fixing and tracking a target comprising at least one such contrasting edge," FR Patent EP2 341312(A1) and WO2 $011073079(A 1), 06$ 23, 2011. [Online]. Available: http://worldwide.espacenet.com/publicationDetails/biblio? $\mathrm{FT}=\mathrm{D} \&$ date $=20110706 \& \mathrm{DB}=\mathrm{EPODOC} \&$ locale $=$ en $\_\mathrm{EP} \& \mathrm{CC}=\mathrm{EP} \& \mathrm{NR}=$ $2341312 \mathrm{~A} 1 \& \mathrm{KC}=\mathrm{A} 1$

[43] C. Blanès, "Appareil visuel élémentaire pour la navigation à vue d'un robot mobile autonome," Master's thesis, DEA de Neurosciences, Université de la Méditerranée (Aix-Marseille II), Marseille, 1986.

[44] F. Ruffier, S. Viollet, S. Amic, and N. Franceschini, "Bio-inspired optical flow circuits for the visual guidance of micro air vehicles," in Circuits and Systems, 2003. ISCAS '03. Proceedings of the 2003 International Symposium on, vol. 3, 2003, pp. 846-849.

[45] M. Pudas, S. Viollet, F. Ruffier, A. Kruusing, S. Amic, S. Leppavuori, and N. Franceschini, "A miniature bio-inspired optic flow sensor based on low temperature co-fired ceramics (ltcc) technology," Sensors and Actuators A: Physical, vol. 133, no. 1, pp. 88-95, Jan. 2007. [Online]. Available: http://www.sciencedirect.com/science/article/ B6THG-4JX9V7Y-1/1/44261a5d214b8ba6b901aa31f96a6854

[46] S. Viollet, F. Ruffier, T. Ray, L. Kerhuel, F. Aubépart, N. Franceschini, and M. Ménouni, "Performances of three miniature bio-inspired optic flow sensors under natural conditions," Sensors \& Transducers Journal, vol. 9, pp. 151-159, December 2010.

[47] L. Kerhuel, S. Viollet, and F. Ruffier, "Method and device for measuring the angular velocity of a luminance transition zone and steering aid system for fixation and tracking a target comprising at least one such luminance transition zone," FR Patent EP2 341 352(A1) and WO2 $011073085(\mathrm{~A} 1), 06$ 23, 2011. [Online]. Available: http://worldwide.espacenet.com/publicationDetails/biblio? $\mathrm{FT}=\mathrm{D} \&$ date $=20110706 \& \mathrm{DB}=\mathrm{EPODOC} \&$ locale $=$ en_EP $\& C C=\mathrm{EP} \& \mathrm{NR}=$ $2341352 \mathrm{~A} 1 \& \mathrm{KC}=\mathrm{A} 1$

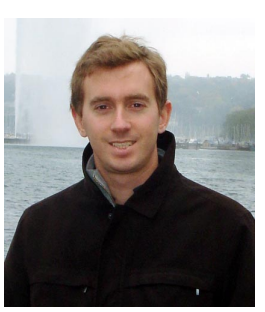

Lubin Kerhuel was born and raised in the Paris region of France. He obtained a Bachelor in Science degree in System-on-Chip from ESIEE Paris, and a Master in Science degree in Signal Processing and Numerical Communication from the University of Nice Sophia Antipolis. He then obtained a doctorate degree in Control Engineering and Microelectronics from the University of Montpellier II. He conducted his $\mathrm{Ph} . \mathrm{D}$ research on visual sensors and micro-aerial vehicle adapted control laws within the Biorobotics Laboratory, Institute of Movement Sciences, National Center for Scientific Research, at the University Aix-Marseille. He is an expert in rapid prototyping technology and developed a Simulink blockset to target Microchip microcontrollers, used worldwide. He is currently developing a miniature, low power, movement and attitude reconstruction inertial movement unit, based on Microelectromechanical systems (MEMS) at MOVEA, in Grenoble, France.

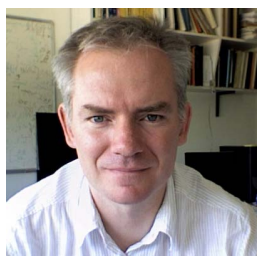

Stéphane Viollet was born in Limoges, France. $\mathrm{He}$ received the Master's degree in control engineering from the University of Bordeaux 1, Bordeaux, France, and the Ph.D. degree from the National Polytechnic Institute, Grenoble, France, in September 2001. He is currently the head of the Biorobotics Dpt., Institute of Movement Sciences, National Center for Scientific Research/University of the Mediterranean, Marseille, France. His current research interests include biorobotics, oculomotor control, and retinal micromovements, as well as the development of novel bioinspired visual sensors and control laws for implementation onboard autonomous flying robots.

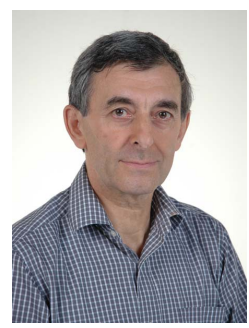

Nicolas Franceschini was born in Mâcon, France. He graduated in electronics and control theory from the National Polytechnic Institute, Grenoble. He then switched to neuroscience and behavioral science, spending 13 years at the Max-Planck Institute for Biological Cybernetics, Tübingen, Germany. In 1972, he received the Dr es Sciences in Physics from the University of Grenoble. Back to France in 1979, he created the Neurocybernetics Laboratory - and later the Biorobotics Laboratory - at CNRS, Marseille. His research interests cover neural information processing, vision, eye movements and retinal micromovements, micro-optics, neuromorphic circuits, sensory-motor control systems, and their potential applications to micro- air- and space- vehicles. He received several national and international awards in both Neuroscience and Engineering, and is a member of the Academia Europaea. 